\title{
Relación entre autoconcepto y perfiles de resiliencia en jóvenes con discapacidad
}

\author{
Raquel Suriá Martínez
}

Departamento de Comunicación y Psicología Social, Universidad de Alicante

\section{España}

Correspondencia: Raquel Suriá Martínez. Departamento de Comunicación y Psicología Social. Edificio de Cienncias Sociales, Universidad de Alicante. Carretera San Vicente del Raspeig s/n. 03690 San Vicente del Raspeig - Alicante. España. E-mail: raquel.suria@ua.es

(C) Education \& Psychology I+D+i and Ilustre Colegio Oficial de la Psicología de Andalucía Oriental (Spain) 


\section{Resumen}

Introducción. En este trabajo se analiza el autoconcepto y la resiliencia en un grupo de jóvenes con discapacidad según la tipología y grado de discapacidad. Asimismo, se examina si existen combinaciones de los componentes de resiliencia que den lugar a diferentes perfiles resilientes. Finalmente, se comprueba si existen diferencias estadísticamente significativas entre los grupos obtenidos respecto al autoconcepto.

Método. La Escala de Autoconcepto F5 y la Escala de Resiliencia fueron administradas a una muestra de 114 jóvenes con diferentes tipos de discapacidad (física, intelectual, visual y auditiva). El rango de edad fue 18 a 35 años $(M=26,22 ; D T=4,02)$.

Resultados. El análisis de conglomerados identificó tres perfiles resilientes. Entre estos perfiles se encontraron diferencias estadísticamente significativas respecto a las dimensiones de autoconcepto.

Discusión y conclusión. Esto sugiere la importancia de diseñar programas que potencien la resiliencia, con objeto de desarrollar el autoconcepto entre los jóvenes con discapacidad.

Palabras Clave: jóvenes con discapacidad; diversidad funcional; capacidad resiliente; autoconcepto; programas de intervención. 


\begin{abstract}
Introduction. The present study is aimed to identify different profiles in self concept and the resilience. In addition, significant differences in self-concept domains among profiles previously identified are also analyzed.
\end{abstract}

Method. AF5 Self-concept Questionnaire [Cuestionario de Autoconcepto AF5 and the Resilience Scale were administered to 114 young people with different disabilities (physical, intellectual, visual and auditory), 18 to 35 years $(M=26,22 ; D T=4,02)$.

Results. Cluster analyses allowed identifying three different resilience profiles. Results also revealed significant statistical differences in resilience among the participants based on the type of disability. This was also observed in most domains of self-concept among profiles.

Discussion y Conclusion. Results suggest the need of going in depth on resilience knowledge and design self-concept programs for of people with disabilities.

Keywords: Young people with disabilities; Functional diversity; Resilient capacity; Selfconcept; Physical disability; Intervention programs. 


\section{Introducción}

El interés por potenciar el autoconcepto de los jóvenes con discapacidad se ha incrementado notablemente en los programas de integración de este colectivo (Antle, 2004; Guest, Klose, Needham-Shropshire y Jacobs, 1997; Pérez y Garaigordobil, 2007; Sánchez y LópezJusticia, 2012; Sherer et al., 1982). Este constructo se considera una de las variables más importantes que se encuentran presentes en el individuo, al estar conformada principalmente por las referencias, sentimientos y las ideas que se tienen de uno mismo, lo cual permite dirigir la vida desde una perspectiva tanto académica/profesional y social, como personal (Fuentes, García, Gracia y Lila, 2011; García, Musitu, Riquelme y Riquelme, 2011; García, Gracia y Zeleznova, 2013; Goñi, Fernández-Zabala e Infante, 2012).

\section{Autoconcepto y discapacidad}

Así, el autoconcepto sería el encargado de crear un marco de referencia para interpretar la realidad externa y las propias experiencias, condicionando las expectativas y la motivación, y de esta forma, contribuyendo a la salud, al equilibrio psíquico (García, Musitu, Riquelme y Riquelme, 2011) y al bienestar subjetivo de la persona (Polo-Sánchez y LópezJusticia, 2012). Todo esto, que es sustancial para todos los individuos, cobra especial relevancia en los colectivos más vulnerables, como sucede con los jóvenes que viven la experiencia de tener una discapacidad (Morales, Fernández, Infante, Trianes y Cerezo, 2010).

Si se atiende a las cifras de la Encuesta sobre discapacidad, autonomía personal y situaciones de dependencia (EDAD), realizada por el Instituto Nacional de Estadística (2008), se estima que en España viven 163.650 personas con discapacidad entre 15 y 30 años. En este sentido, la Clasificación Internacional del Funcionamiento, de la Discapacidad y de la Salud (OMS, 2001) estableció que la discapacidad es el resultado de la interacción de la persona que presenta una deficiencia ante las barreras físicas y actitudinales de su entorno, conformando por tanto las actitudes negativas y los prejuicios, importantes barreras para su inclusión social (de Boer, Pijl y Minnaert, 2010; Novo-Corti, Muñoz-Cantero y Calvo-Porral, 2011; Suriá, 2014). 
En este sentido, no es únicamente la actitud del resto de la sociedad la determinante de la integración de las personas con discapacidad, sino que la percepción que se tiene sobre uno mismo, es decir, el autoconcepto, el que tiene un papel primordial, puesto que éste determina las metas y expectativas, guiando la conducta de la persona (Polo-Sánchez y López-Justicia, 2012). De esta forma, es muy probable que si una persona se ve a sí misma de forma negativa, el resultado será una imagen y un trato menos desfavorables por parte de los demás que si se tiene una visión positiva de sí misma (Morales et al., 2010). En relación a esto, diferentes estudios (Buscaglia,1990; Pérez y Garaigordobil, 2007; Polo-Sánchez y López-Justicia, 2012), indican que, aunque el autoconcepto de las personas con discapacidad crece y se desarrolla del mismo modo que el de las personas sin discapacidad, su evolución se ve mermada con mayor frecuencia debido a que la persona con discapacidad recibe influencias negativas, enfrentándose desde la infancia, en muchos casos, al rechazo social y a experiencias negativas en las relaciones interpersonales, que lo desvalorizan y frustran. Estas circunstancias desembocan en una mayor probabilidad de desarrollar un autoconcepto negativo y por tanto, en la necesidad de incidir en un colectivo como es el de los jóvenes con discapacidad, colectivo que se encuentra en situación de riesgo, en primer lugar, por la experiencia de vivir con discapacidad (Martínez, García y Pérez, 2005; Matalinares, et al., 2011; Suriá, García-Fernández y Ortigosa, 2015), y en segundo lugar, por la etapa de la vida en la que se encuentran, período evolutivo del ciclo vital caracterizado por una mayor diferenciación del autoconcepto ya que, los jóvenes se enfrentan a nuevos roles sociales y cognitivos, así como a importantes cambios físicos y corporales que propician la aparición de nuevas dimensiones de autovaloración. Todos estos cambios, junto a una mayor vulnerabilidad o susceptibilidad de los jóvenes para distorsionar su propia imagen, determinan el enorme interés que despierta el estudio del autoconcepto en este periodo del ciclo vital (Esnaola, 2009; Luna y Molero, 2013; Saavedra y Villalta, 2008).

\section{Resiliencia y discapacidad}

En este sentido, numerosas investigaciones han examinado diferentes variables que pueden estar involucradas con el desarrollo del autoconcepto, destacándose la inteligencia emocional (Rey y Extremera, 2012), la conducta prosocial (Luna y Molero, 2013) el empoderamiento (Silva-Dreyer y Martínez-Guzmán, 2007) o la resiliencia (Matalinares et al., 2013) entre algunas de ellas. Centrándonos en ésta última, la resiliencia, la evidencia empírica ha puesto de manifiesto que ésta, es uno de los factores más determinante en la integración y calidad de vida en general, de las personas con discapacidad (Gil-Llario, Molero-Mañes, Ba- 
llester-Arnal y Sabater-Pavía, 2012; Gross-Fava y Tomba, 2009; Quiceno Sierra y Vinaccia, 2012; Saavedra y Villalta, 2008; Suriá, 2014). Este término fue acuñado por Rutter (1972), y se caracteriza como la capacidad de una persona de sobreponerse a la adversidad, recuperarse y salir fortalecido de ella, permitiendo desarrollar competencias sociales, académicas y vocacionales, pese a estar expuesto a situaciones de estrés y dificultad grave (Grotberg, 1995). De esta definición se desprende que la resiliencia, es un conjunto de procesos sociales e intrapsíquicos que tienen lugar a través del tiempo, dando combinaciones entre atributos de la persona y su ambiente, social y cultural, tratándose por tanto, de un proceso interactivo conformado por diferentes dimensiones (Kotliarenco, 2000; Saavedra y Villalta, 2008; Uriarte, 2013).

Tras una revisión entre la literatura sobre este constructo, y aunque parece que no existe unanimidad al delimitar el número de dimensiones que componen esta capacidad (Heilemann, Lee y Kury, 2003; Oshio, Nakaya, Kaneko y Nagamine, 2002; Rodríguez et al., 2009; Salgado, 2005; Wagnild y Young, 1993, por citar algunos), sí se está de acuerdo en indicar que las personas resilientes son definidas como personas socialmente competentes, que tienen conciencia de su identidad, que pueden tomar decisiones, establecer metas y creer en un futuro mejor, satisfacer sus necesidades básicas de afecto, relación, respeto y la consecución de sus metas (Rybarczyk, Emery, Guequierre, Shamaskin y Behel, 2012; Saavedra y Villalta, 2008). Asimismo, existe acuerdo en definir este concepto como un conjunto de procesos sociales e intrapsíquicos que tienen lugar a través del tiempo, dando combinaciones entre atributos de la persona y su ambiente, social y cultural, tratándose por tanto, de un proceso dinámico en el que interactúan las diferentes dimensiones que componen el constructo (Kotliarenco, 2000; Saavedra y Villalta, 2008; Uriarte, 2013; Vinaccia, Quiceno y Moreno San Pedro, 2007).

Entre las dimensiones que conforman la resiliencia se encuentran la Aceptación de la vida y de uno mismo, la Competencia Social y la Autodisciplina. Si retomamos el concepto de autoconepto y las dimensiones que lo componen, se puede encontrar cierta similitud en algunas de éstas, como la Autoaceptación y las Relaciones positivas con los demás y los componentes citados de la resiliencia. Por tanto, es posible que exista una vinculación directa entre los componentes de ambos constructos, esto es, entre el autoconcepto y la resiliencia. Por otra parte, si ambos constructos están compuestos por diferentes factores, esto podría implicar 
que cada una de los componentes de resiliencia no tengan la misma relevancia en el autoconcepto.

\section{Autoconcepto y resiliencia en la discapacidad}

En relación a la literatura publicada sobre el autoconcepto y su posible asociación con la resiliencia en jóvenes con diferentes tipos de discapacidad, en la actualidad no se han encontrado trabajos previos que analicen en profundidad la relación de ambos constructos, y que a su vez, analicen la influencia de la tipología y grado de severidad de la discapacidad en el autoconcepto y la resiliencia. Centrándonos en la resiliencia, en líneas anteriores se ha enfatizado en la importancia de fomentar este constructo entre los personas con discapacidad, puesto que ésta promueve el bienestar emocional, el desarrollo personal, la inclusión social y la calidad de vida (Gifre, Del Valle, Yuguero, Gil y Monreal, 2010; Saavedra y Villalba, 2008; Suriá, 2014; Suriá et al., 2015), sin embargo, al atender a la litertura sobre esta tematica, son inexistentes los estudios enfocados a analizar la existencia de combinaciones de los componentes de resiliencia (Aceptación de la vida y de uno mismo, Competencia Social y Autodisciplina), que den lugar a diferentes perfiles de este constructo entre estos jóvenes. Finalmente, desde este trabajo se desconocen estudios publicados sobre diferencias en autoconcepto entre distintos perfiles resilientes de jóvenes con diferentes tipos de discapacidad, que tengan en cuenta, no sólo al autoconcepto general sino también a otras dimensiones del autoconcepto (e.g., autoconcepto académico/laboral, social, emocional, etc.).

\section{Objetivos}

A partir de estas consideraciones, en el presente estudio se plantean tres objetivos. Primero: conocer el autoconcepto y la resiliencia en una muestra de jóvenes con discapacidad. Esto se analizará en función de la tipología y del grado de severidad de la discapacidad. Segundo: identificar si entre los participantes existen combinaciones de diferentes dimensiones de resiliencia que den lugar a diferentes perfiles, los cuales podrían definirse en función del peso que tengan cada una de las dimensiones dentro de cada perfil. Tercero: una vez hallados y definidos los perfiles de resiliencia, se analiza si existen diferencias estadísticamente significativas entre los perfiles de esta fortaleza definidos y las diferentes puntuaciones en autoconcepto. 


\section{Método}

\section{Participantes}

La investigación se llevó a cabo sobre una muestra intencional -por motivos de accesibilidad- de 116 jóvenes con discapacidad, todos ellos procedentes de diversas asociaciones dedicadas a personas con discapacidad. Inicialmente, la población de estudio estaba conformada por 132 jóvenes, sin embargo, de estos, fueron 114 los que se dispusieron a participar (véase tabla 1), correspondiendo el 53,5\%, al género femenino y el 46,5\% al masculino, con edades comprendidas entre 18 y 37 años $(M=28,22 ; D T=4,02)$. Fueron clasificados en función de la tipología de la discapacidad que tenían: el 32,5\%, tenía discapacidad motora, el $21,9 \%$ discapacidad intelectual, el 22,8\%, discapacidad visual y el 22,8\% discapacidad auditiva, y a su vez, según el grado o severidad de la discapacidad, se observa que un $36.8 \%$ tenían más del $65 \%$ de grado de discapacidad, el 38,6\% entre el 33 y el 65\% y el 24,6\%, menos de un $33 \%$ de grado de discapacidad. Con respecto a la etapa en la que adquirieron la discapacidad, siendo el $42,10 \%$ personas con discapacidad adquirida desde el nacimiento y el 57,90\% con discapacidad sobrevenida.

Tabla 1. Perfil sociodemográfico

\begin{tabular}{llll}
\hline Perfil sociodemográfico & $\mathrm{N}$ & $\%$ \\
\hline Sexo & Mujer & 61 & 53,5 \\
& Varón & 53 & 46,5 \\
Edad & $18-22$ & 19 & 16,7 \\
& $23-27$ & 40 & 35,1 \\
& $28-32$ & 32 & 28,1 \\
\multirow{2}{*}{ Tipo de discapacidad } & $33-37$ & 23 & 20,2 \\
& Intelectual & 25 & 21,9 \\
& Auditiva & 26 & 22,8 \\
\multirow{4}{*}{ Grado de discapaci- } & Menos del 33\% & 28 & 32,5 \\
dad & Motora & 37 & 22,8 \\
\multirow{2}{*}{ Etapa de adquisición } & Nacimiento & 48 & 24,6 \\
de discapacidad & Del 33\% al 65\% & 44 & 38,6 \\
& Sobrevenida & 66 & 36,8 \\
\cline { 2 - 4 } & Total & 114 & 42,1 \\
& & & 57,9 \\
\hline
\end{tabular}




\section{Instrumentos}

Cuestionario sociodemográfico; se diseñó un cuestionario ad hoc para la recogida de los datos sociodemográficos: género, edad, tipología y grado de severidad de la discapacidad de los participantes.

Escala de Resiliencia de Wagnild y Young (1993). En su versión adaptada puntúan en una escala tipo Likert de 7 puntos $(1=$ totalmente en desacuerdo, y un máximo de $7=$ totalmente de acuerdo). Las puntuaciones más altas son indicadores de mayor resiliencia, encontrándose el rango de puntaje entre 25 y 175 puntos. Para determinar el nivel de Resiliencia prevalente entre los participantes, se siguió el mismo procedimiento realizado por Heilemann, Lee y Kury (2003). Los valores mayores de 147 indicarían una alta capacidad de resiliencia; entre 121-146, resiliencia moderada; y valores menores a 121, escasa resiliencia. Se eligió esta prueba por su fácil aplicación, por estar validada para población joven y adulta y finalmente, por las propiedades psicométricas que muestra tanto la versión original (Wagnild y Young, 1993) como su versión en castellano (Heilemann et al., 2003), mostrándose unos índices de consistencia interna elevados en ambas versiones $(\alpha=, 89$ y el $\alpha=, 93$ respectivamente). En el presente estudio, el análisis factorial exploratorio mostró una varianza explicada del $81,20 \%$ distribuida en tres factores diferenciados:

Factor 1. Competencia personal, entendida como el reconocimiento de factores de capacidad personal, independencia, dominio, perseverancia, habilidad, etc. Este factor está conformado por 13 reactivos y explica el $35,37 \%$ de varianza,

Factor 2. Aceptación de sí mismo y de la vida, como sinónimo de adaptación, flexibilidad, etc., compuesto por 6 reactivos restantes que explican el 24,42\% de varianza y,

Factor 3. Autodisciplina, con una carga factorial del 21,40\% y conformado por los 6 ítems restantes. Aunque en la versión original el instrumento muestra dos factores, en este trabajo, y al igual que han evidenciado otros autores trabajos que utilizaron el instrumento original (Rodríguez et al., 2009; Suriá, 2012; Vara y Rodríguez, 2011), se obtiene un tercer factor acuñado con el nombre de "Autodisciplina". Asimismo la consistencia interna de la escala con base al índice alfa de Cronbach es satisfactoria $(\alpha=, 88)$.

Escala de Autoconcepto Forma 5 (AF-5), de García y Musitu (1999). El AF5 parte de una consideración multidimensional del autoconcepto, perspectiva que tiene en la actualidad 
mayor apoyo empírico. Este instrumento se fundamenta en el modelo teórico de Shavelson et al. (1976), y consta de 30 ítems distribuidos en cinco dimensiones: el académico/laboral (ítems 1, 6, 11, 16, 21 y 26), social (ítems 2, 7, 12, 17, 22 y 27), emocional (ítems 3, 8, 13, 18, 23 y 28), familiar (ítems 4, 9, 14, 19, 24 y 29) y físico (ítems 5, 10, 15, 20, 25 y 30); es decir, seis ítems por cada una de ellas y los participantes tienen que responder a los ítems en una escala tipo Likert, ordenadas desde aquella con una connotación más positiva hasta la más negativa (donde $1=$ totalmente en desacuerdo y $5=$ totalmente de acuerdo). Así, el rango de las puntuaciones posibles del cuestionario tiene un puntaje general mínimo de 30 y un máximo de 150 (cuanto mayor es la puntuación, mayor es el autoconcepto).

Se eligió esta escala por haber sido utilizada en otros estudios con personas que tenían similares características a las del presente trabajo (Gómez-Vela, Verdugo y González-Gil, 2007); es fácil de administrar y se puede aplicar a niños y adultos con diferente nivel académico; asimismo, la estructura factorial de los ítems de la escala confirmó satisfactoriamente las dimensiones teóricas, los componentes explicaban el 51\% de la varianza total (coeficiente alpha de Cronbach de ,84). Con respecto a las propiedades psicométricas de la escala para el presente estudio, el análisis de consistencia interna indicó una fiabilidad adecuada $(\alpha=, 76)$, asimismo, la varianza explicó el 60,04\% de la variabilidad.

\section{Procedimiento}

Se trata de un tipo de estudio de serie de casos transversal. Para recoger los datos se aplicaron las escalas a los participantes. La muestra procedía de diversas asociaciones. Así, para solicitar la colaboración de las personas con discapacidad motora se acudió a la federación de ASPAYM (Asociación de Parapléjicos y Personas con Gran Discapacidad Física). Para los participantes con discapacidad auditiva se acudió a APSA (Asociación prodiscapacitados psíquicos de Alicante (España). Con objeto de recopilar datos de personas con discapacidad auditiva se acudió a APANAH (Asociación de Padres y Deficientes Auditivos de Alicante). Finalmente, se acudió a la ONCE (Organización Nacional de Ciegos Españoles), para solicitar colaboración a las personas con discapacidad visual.

Tras un primer contacto con los directivos de las entidades para explicarles el objetivo del estudio, se propuso la colaboración a los participantes. Para ello, se acudió a las reuniones que se realizan periódicamente en estas asociaciones y se les administró, voluntaria, anónima -459 -

Electronic Journal of Research in Educational Psychology, 14(3), 450-473. ISSN: 1696-2095. 2016. no. 40 http://dx.doi.org/10.14204/ejrep.40.15150 
y presencialmente los cuestionarios, una vez que los participantes otorgaron su consentimiento por escrito. Y las personas con discapacidad intelectual, cómo fue el consentimiento?? La aplicación del cuestionario se adaptó a las condiciones de cada participante. El tiempo estimado de aplicación fue aproximadamente de 30 minutos para ambos cuestionarios. Los evaluadores fueron previamente entrenados en la aplicación del instrumento. La recopilación de datos se llevó a cabo entre marzo y diciembre del 2014.

\section{Análisis de datos}

Para los datos sociodemográficos se obtuvieron las frecuencias y porcentajes. Para comprobar si existían diferencias estadísticamente significativas en autoconcepto y en resiliencia en función de la tipología y del grado de severidad de la discapacidad se utilizó el análisis de varianza (ANOVA). Previo a esto, se comprobó que se cumplían los supuestos de homocedasticidad de varianza, normalidad de las distribuciones e independencia de variables. Esto se comprobó a través de la prueba de Levene, de Kolmogorov-Smirnov y Chi cuadrado respectivamente. Asimismo, se calculó el tamaño del efecto (diferencia media tipificada o índice $d$; Cohen, 1988), el cual indica si la o magnitud de las diferencias halladas es pequeña, moderada o grande.

Para identificar los perfiles de resiliencia se utilizó el método de análisis cluster en dos etapas (2-step cluster analysis), es una herramienta exploratoria diseñada para revelar las agrupaciones naturales dentro de un conjunto de información que, de otra manera, no sería evidente. Asimismo, el procedimiento puede determinar automáticamente el número óptimo de clusters. Los perfiles se han definido a partir de las diferentes combinaciones de las tres dimensiones de resiliencia que evalúa la Escala de Resiliencia de Wagnild y Young (1993): Competencia personal y Aceptación de sí mismo y de la vida y Autodisciplina.

Posteriormente, se realizaron análisis de varianza (ANCOVA) en los clusters obtenidos con el objetivo de analizar la significación estadística de las diferencias existentes entre los grupos en los factores de Autoconcepto. Se controló la covariante edad para controlar su posible influencia en los resultados. Finalmente, se realizaron pruebas post hoc para identificar entre qué grupos se encontraban las diferencias. Se utilizó el método de Scheffé al no estar cada grupo compuesto por el mismo número de participantes. Para analizar la magnitud o tamaño del efecto de estas diferencias, se atendió al índice directo de eta ${ }^{2}$. Asimismo, se calculó el tamaño del efecto (diferencia media tipificada o índice $d$; Cohen, 1988) para calcular 
la magnitud de las diferencias observadas. Los datos se analizaron por medio del paquete estadístico SPSS versión 19.0.

\section{Resultados}

\section{Autoconcepto y Resiliencia en función de la tipología y grado de discapacidad}

Al examinar las puntuaciones medias de autoconcepto entre los grupos, se observó que los participantes presentaron puntuaciones moderadas en la escala. No se encontraron diferencias estadísticamente significativas ni según la tipología $\left[F_{(3,110)}=0,35, p=, 986\right]$, ni el grado de severidad de la discapacidad $\left.\left[F_{(2,111}\right)=1,96, p=, 148\right]$.

En lo que respecta a la resiliencia, se encontró un nivel elevado de esta capacidad entre los participantes, indicándose en los resultados al examinar la resiliencia en función del tipo de discapacidad, unos niveles más elevados en los jóvenes con discapacidad visual y en los participantes con discapacidad motora, $\left[F_{(3,110)}=3,859, p=, 042\right]$, siendo el tamaño del efecto elevado en el análisis post hoc entre el grupo de participantes con discapacidad visual $(d=$ $0,69)$ y el grupo de jovenes con discapaccidad auditiva e intlectual $(d=0,72)$ así como entre la comparación del grupo con discapacidad motora con el grupo con discapacidad auditiva ( $d$ $=0,57)$ e intelectual $(d=0,54)$. Sin embargo, no se encontraron diferencias estadísticamente significativas en función del grado de severidad de la discapacidad, $F_{(2,111)}=1,713, p=, 186$.

Tabla 2. Medias y desviaciones típicas obtenidas en autoconcepto y en resiliencia según tipología y grado de severidad de la discapacidad

\begin{tabular}{lcccc}
\hline Tipo/grado de & \multicolumn{2}{c}{ Autoconcepto } & \multicolumn{2}{c}{ Resiliencia } \\
\cline { 2 - 5 } discapacidad & $M$ & $D T$ & $M$ & $D T$ \\
\hline Intelectual & 103,05 & 20,62 & 123,90 & 40,62 \\
Auditiva & 100,43 & 19,35 & 123,43 & 22,80 \\
Motora & 97,83 & 26,23 & 148,97 & 23,35 \\
Visual & 101,94 & 23,49 & 153,33 & 14,86 \\
Total & 101,66 & 25,58 & 136,46 & 30,54 \\
$F$ & \multicolumn{3}{c}{0,35} & \multicolumn{2}{c}{$3,859 *$} \\
Menos del 33\% & 101,50 & 27,83 & 141,09 & 27,77 \\
Del 33\% al 65\% & 95,32 & 25,34 & 138,07 & 26,61 \\
Más del 65\% & 93,29 & 22,51 & 130,19 & 34,83 \\
Total & 105,66 & 25,58 & 136,33 & 30,44 \\
$F$ & \multicolumn{3}{c}{1,960} \\
**) Nivel de Significación de ,001; (*) = Nivel de Significación de ,05.
\end{tabular}




\section{Identificación de perfiles de resiliencia}

El método cluster, tratando de lograr la máxima homogeneidad en cada grupo y las mayores diferencias entre ellos, determinó 3 agrupaciones de las dimensiones de resiliencia. De esta forma, la resiliencia quedó integrada por un grupo (BC-BA-BA) formado por 28 participantes (24,56\%), caracterizado por bajas puntuaciones en las 3 dimensiones de resiliencia. El segundo cluster (AC-AA-BA), integrado por 41 participantes $(35,96 \%)$ compuesto por puntuaciones altas en Competencia personal y Aceptación de uno mismo y bajas en Autodisciplina. Finalmente, el último cluster (AC-AA-AA) conformado por 45 personas $(39,47 \%)$, caracterizado por un predominio de altas puntuaciones en las 3 dimensiones de la escala de resiliencia.

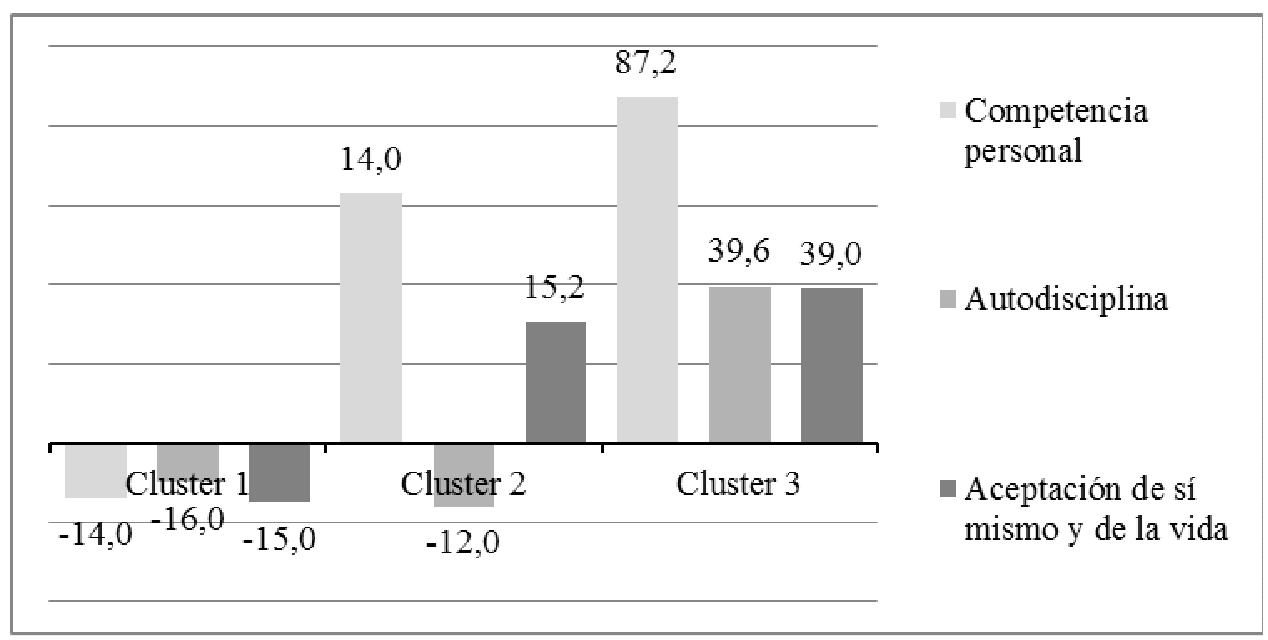

Figura 1. Representación gráfica del modelo de tres clusters: Cluster 1: BC-BA-BA (Baja resiliencia), Cluster 2: AC-AA-BA (Alta Competencia, Baja Autodisciplina y Alta Aceptación), Cluster 3: AC-AA-AA (Alta resiliencia)

\section{Diferencias inter-grupo en dimensiones del autoconcepto}

Los resultados indicaron la existencia de diferencias significativas en función de los clusters resilientes $(\lambda=0,540, F=5,779, p<, 000)$. No se observó influencia de la covariante $\operatorname{edad}(\lambda=0,097, F=2,100, p=, 978)$.

$\mathrm{Al}$ atender a las puntuaciones medias de la escala global de autoconcepto se observaron diferencias estadísticamente significativas en los tres grupos o clusters $\left(F_{(2,111)}=7,34, p<\right.$ 
,05, $\eta^{2}=, 10$ ), encontrándose que el Grupo 3 (AC-AA-AA) indicó medias superiores al Grupo 2 (AC-AA-BA), $(d=0,11)$ y al Grupo 1 (BC-BA-BA), $(d=0,90)$ en autoconepto global. Asimismo, el Grupo 2 (AC-AA-BA) mostró puntuaciones medias más elevadas que el Grupo 1 $(\mathrm{BC}-\mathrm{BA}-\mathrm{BA}),(d=0,71)$.

$\mathrm{Al}$ examinar los diferentes factores que componn el autoconcpto y las comparaciones post hoc para ver entre que grupos se encontraban las diferencias se observaron los siguientes resultados:

Con respecto al Factor 1: Autoconcepto Académico/laboral, que el Grupo de altas puntuaciones en las tres dimensiones de resiliencia, es decir, e1 Grupo 3 (AC-AA-AA), indicó tener medias significativamente superiores que el Grupo 2 (AC-AA-BA) y que el Grupo 1 (BC-BA-BA), $\left(F_{(2,111)}=4,49, p<, 05, \eta^{2}=, 04\right)$, siendo el tamaño del efecto moderado en el análisis post hoc entre el Grupo 3 (AC-AA-AA) y el Grupo 2 (AC-AA-BA), $(d=0,21)$ y elevado entre la comparación del Grupo 3 (AC-AA-AA) con el Grupo 1 (BC-BA-BA), $(d=$ 0,73). A su vez, el Grupo 2 (AC-AA-BA), mostró puntuaciones más elevadas que el Grupo 1 (BC-BA-BA), $(d=0,55)$.

En lo relativo al Factor 2, Autoconcepto social, se observaron diferencias estadísticamente significativas entre los clusters, $\left(F_{(2,111)}=3,79, p<, 05, \eta^{2}=, 03\right)$, encontrándose que el grupo que señaló puntuaciones elevadas en las tres dimensiones de resiliencia, esto es, el Grupo 3 (AC-AA-AA) así como los grupos en los que las puntuaciones medias fueron elevadas en Competencia social y en Aceptación de uno mismo y de la vida, Grupo 2 (AC-AABA) mostraron puntaciones más elevadas en el Autoconcepto social que el Grupo con bajas puntuaciones en estas dimensiones, Grupo 1 (BC-BA-BA). Así, el Grupo 3 indicó medias significativamente superiores al compararlo con el Grupo $1(d=0,84)$. Asimismo, el Grupo 2 (AC-AA-BA) indicó puntuaciones medias superiores al Grupo 1 (BC-BA-BA), $(d=0,54)$ en este factor de autoconcepto.

La misma dirección se encontró en el Factor 3, relativo al Autoconcepto Familiar y en el Factor 4, concerniente con el Autoconcepto Emocional. Así, en el factor referente a la familia (Factor 3), se observó que el Grupo 3 (AC-AA-AA), indicó puntuaciones más elevadas 
que el Grupo 1 (BC-BA-BA), $\left(F_{(2,111)}=17,55, p<, 001, \eta^{2}=, 34\right),(d=1,24)$ y que el Grupo 2 (AC-AA-BA), $(d=0,82)$ en autoconcepto familiar. De igual manera, el Grupo 2 (AC-AABA) indicó medias superiores al Grupo 1(BC-BA-BA), $(d=0,62)$. En lo concerniente al Factor 4, Autoconcepto emocional, también se observaron diferencias estadísticamente significativas entre los tres clusters, destacando el Grupo 3 (AC-AA-AA), con puntuaciones medias superiores al Grupo 1 (BC-BA-BA), $\left(F_{(2,111)}=9,50, p<, 001, \eta^{2}=, 40, d=0,90\right)$, y puntuaciones medias del Grupo 2 (AC-AA-BA), superiores a las del Grupo 1 (BC-BA-BA), $(d=$ $0,87)$.

Tabla 3. Medias y desviaciones típicas obtenidas por los tres grupos y valores de "eta cuadrado $\left(\eta^{2}\right)$ para cada una de las dimensiones del autoconcepto

\begin{tabular}{|c|c|c|c|c|c|c|c|c|c|c|c|}
\hline \multirow[t]{2}{*}{$\begin{array}{l}\text { Factores de } \\
\text { autoconcepto }\end{array}$} & \multicolumn{2}{|c|}{$\begin{array}{c}\text { Grupo 1 } \\
\text { (BC-BA-BA) }\end{array}$} & \multicolumn{2}{|c|}{$\begin{array}{c}\text { Grupo } 2 \\
\text { (AC-AA-BA) }\end{array}$} & \multicolumn{2}{|c|}{$\begin{array}{c}\text { Grupo } 3 \\
\text { (AC-AA-AA) }\end{array}$} & \multicolumn{2}{|c|}{ Total } & \multirow[t]{2}{*}{$F$} & \multirow[t]{2}{*}{$p$} & \multirow[t]{2}{*}{$\eta^{2}$} \\
\hline & $M$ & $D T$ & $M$ & $D T$ & $M$ & $D T$ & $M$ & $D T$ & & & \\
\hline Académico/laboral & 22,20 & $(5,69)$ & 25,45 & $(5,98)$ & 26,00 & $(4,52)$ & 24,14 & $(5,92)$ & 4,49 & ,014 &, 36 \\
\hline Social & 20,39 & $(4,56)$ & 24,57 & $(5,28)$ & 22,80 & $(4,48)$ & 20,45 & $(5,01)$ & 3,79 & ,047 & ,28 \\
\hline Familiar & 21,07 & $(4,59)$ & 27,79 & $(6,13)$ & 23,60 & $(3,75)$ & 24,55 & $(6,17)$ & 17,55 &, 000 &, 52 \\
\hline Emocional & 21,32 & $(6,10)$ & 27,21 & $(7,11)$ & 26,00 & $(4,52)$ & 24,62 & $(7,02)$ & 9,50 &, 000 &, 40 \\
\hline Físico & 21,51 & $(5,72)$ & 22,11 & $(4,86)$ & 21,20 & $(5,03)$ & 22,05 & $(5,33)$ & 2,82 &, 064 &, 14 \\
\hline Total & 106,49 & $(23,02)$ & 123,13 & $(23,23)$ & 125,60 & $(19,20)$ & 116,42 & $(24,10)$ & 7,34 &, 001 &, 33 \\
\hline
\end{tabular}

\section{Discusión y conclusiones}

Este trabajo trata de profundizar en la relación entre el autoconcepto y la resiliencia en los jóvenes con diferentes tipos y grados de discapacidad. Para ello se plantean diferentes objetivos. En primer lugar, examina el nivel de autoconcepto y de resiliencia en un grupo de jóvenes con discapacidad, los resultados reflejan que los participantes tienen niveles moderados en ambos constructos. Estos resultados respaldan la idea de otros autores que resaltan el proceso de lucha que ponen en marcha las personas con discapacidad para afrontar con éxito los obstáculos derivados de su discapacidad (Morales et al., 2010; Saavedra y Villalta, 2008; Suriá, 2014).

$\mathrm{Al}$ atender a los resultados de resiliencia en función de la tipología de la discapacidad, los resultados indican que las puntuaciones más altas las mostraron los jóvenes con discapaci- 
dad motora y visual, mientras que desde la discapacidad auditiva e intelectual se revelaron puntuaciones inferiores. Una explicación para estos resultados podría apoyarse en el efecto que genera en la sociedad, la visibilidad de estos dos tipos de discapacidad. Desde hace algunos años, desde diferentes ámbitos sociales y políticos están fomentando el derecho de las personas con discapacidad a la inclusión social y con ello, a que la sociedad tome mayor conciencia en ofrecer apoyo para la plena integración psicosocial hacia las personas que viven con esta problemática (Floyd, Zambrano, Antó, Jiménez, Solórzano y Díaz, 2012; Suriá, 2012, Thompson et al., 2010). Esto se refleja en las discapacidades más visibles, como puede ser una discapacidad motora o una discapacidad visual. De esta manera, independientemente de que la persona con discapacidad utilice las ayudas disponibles, si va a percibir que dispone de apoyo. Por el contrario, existen otras discapacidades que son invisibles como la discapacidad intelectual o la discapacidad auditiva, las cuales pasan desapercibidas en muchos casos por la sociedad, derivando en que el resto de personas no se involucren tanto en ofrecer ayuda y con ello, que la persona con esta problemática perciba más desprotección (Aguado Díaz y Alcedo Rodríguez, 2012; Juárez-Sánchez et al., 2010; Schalock, 2013).

La otra variable tomada en consideración en este primer objetivo es la influencia del grado de discapacidad en el autoconcepto y en la resiliencia. En este sentido, en los resultados no se refleja la influencia de esta variable, respaldando estos datos la definición de resiliencia. Así, si se parte de la propia definición de resiliencia y esta capacidad se desarrolla según el individuo va encontrándose obstáculos y va superándolos, se podría explicar la inexistencia de diferencias entre los grupos en función de la severidad de la discapacidad. Así, las limitaciones a las que se enfrentan las personas que viven esta situación van a propiciar oportunidad para potenciar esta fortaleza, por lo que, un mayor grado de discapacidad puede generar mayor capacidad de superación y con ello, la no diferenciación del grado de resiliencia con los participantes con menor grado de discapacidad. Por otra parte, las personas, independientemente del grado de severidad de su discapacidad, que tienen la iniciativa de acudir en busca de apoyo a grupos o asociaciones de autoayuda, pueden tener mayor predisposición a superar sus barreras, de ahí que la mayoría de estos jóvenes tengan un nivel de resiliencia bastante potencializado.

De manera similar, el autooncepto, no se ve afectado por el grado de discapacidad. En este sentido, desgraciadamente los estereotipos y modas que marca la sociedad alejan mucho - 465 Electronic Journal of Research in Educational Psychology, 14(3), 450-473. ISSN: 1696-2095. 2016. no. 40 http://dx.doi.org/10.14204/ejrep.40.15150 
a las personas con discapacidad de los cánones de la belleza (Matalinares, et al., 2013; Suriá et al., 2015). Esto podría repercutir en unos niveles inferiores de autoconcepto entre los jóvenes.

Con respecto al segundo objetivo, analizar las posibles combinaciones de las dimensiones resilientes de los jóvenes participantes, con el fin de identificar diferentes perfiles resilientes. Así, mediante el análisis de conglomerados se identifican tres perfiles resilientes distintos, un grupo con un perfil de altas puntuaciones en los tres componentes de resiliencia (AC-AA-AA), un segundo grupo con puntuaciones altas en Competencia social y en Aceptación de uno mismo y de la vida y bajas en Autodisciplina (AC-AA-BA) y un tercer perfil con bajas puntuaciones en las tres dimensiones, esto es, en Competencia social, en Autodisciplina y en Aceptación de uno mismo y de la vida (BC-BA-BA)). Estos resultados confirman por tanto la segunda hipótesis planteada relativa a diferencias en los perfiles resilientes en función del peso de las dimensiones de resiliencia. Al atender al número de participantes que componen cada uno de los clusters, los resultados muestran que es el grupo que puntúa alto en las tres dimensiones, es decir, el grupo de altas puntuaciones en resiliencia (AC-AA-AA) el que está conformado por un mayor número de participantes en comparación con el resto de grupos. Esto refleja en primer lugar, que un alto porcentaje de los jóvenes con discapacidad analizados muestra altos niveles de resiliencia en las tres dimensiones. A su vez, el hecho de que se identifiquen los otros dos grupos con diferentes perfiles de resiliencia indica que la discapacidad puede permitir desarrollar patrones diferenciales de resiliencia. Finalmente, se obtuvo un grupo o perfil de baja resiliencia en las tres dimensiones de resiliencia. Este perfil estaría relacionado con déficits en el ajuste psicológico y en la calidad de vida en general y por tanto, con el hecho de que no todas las personas que viven con una discapacidad tengan ese buen ajuste y adaptación a la experiencia de vivir esta situación (Gifré et al., 2010; Saavedra y Villalba, 2008; Suriá et al., 2015).

En referencia al tercer objetivo, los resultados dan respaldo a la tercera hipótesis planteada, es decir, las puntuaciones medias de los clusters hallados revelan diferencias estadísticamente significativas en el autoconcepto. Estos datos, ofrecen consistencia a la validez de la existencia de diferentes perfiles de resiliencia y ayudan a comprender la relación entre resiliencia y el autoconcepto.De este modo, los resultados reflejan que en la mayoría de factores del autoconcepto, destacan los grupos que puntúan elevado en las tres dimensiones de resiliencia así como en los clusters en los que las dimensiones de Competencia social y de Aceptación de uno mismo y de la vida son elevadas. En este sentido, diferentes autores inciden en 
el papel fundamental que la resiliencia cobra en la vida de las personas que experimentan adversidades, como puede ocurrir entre los jóvenes que viven con discapacidad, por tanto, es coherente encontrar relación entre las dimensiones que componen este constructo y el autoconcepto (Gross-Fava y Tomba, 2009; Suriá et al., 2015).

Estos resultados quedan reforzados al examinar el tamaño del efecto, el cual indica que en la mayoría de factores del autoconcepto, la magnitud de estas diferencias es elevada con respecto a los participantes de bajas puntuaciones en las dimensiones que componen la resiliencia. Esto se observa en los factores relativos al desarrollo de habilidades personales (autoconcepto académico/laboral), así como en las relacionadas con la sociabilidad (autoconcepto social y autoconcepto familiar). En estos factores, se observa que los grupos con puntuaciones altas en las dimensiones de resiliencia de Competencia social/personal y Aceptación de uno mismo y de la vida reflejan puntuaciones medias más elevadas. Esto significa que los jóvenes con baja resliencia tendrían mayores dificultades en cuanto a las relaciones sociales y familiares asi como un menor ajuste y adaptación al medio.

En este sentido, la Competencia social/personal es definida como un comportamiento emitido por la persona en el contexto interpersonal que expresa sentimientos, actitudes, opiniones o derechos de un modo adecuado a la situación (Caballo y Verdugo, 2013). Asimismo, la Aceptación de uno mismo y de la vida, hace referencia a la creencia en uno mismo y al reconocimiento de sus propias fuerzas y limitaciones para afrontar las circunstancias vitales de una forma adecuada (Branden, 1995). Si se tienen en cuenta estas definiciones y, al atender al autoconcepto, estudios previos vinculan el constructo con las relaciones interpersonales positivas (Bisquerra-Alcina y Pérez-Escoda, 2012) y con el funcionamiento social (Lacunza y de González, 2011), aspectos que guardan estrecha relación con la Competencia social/personal y la Aceptación de uno mismo (García et al., 2011). Esta relación explicaría los resultados al examinar los factores relativos al autoconcepto académico/laboral y los relacionados con las interacciones sociales como el autoconcepto social y el familiar.

Finalmente, al examinar el factor de autoconcepto físico, se observa que no difiere en los tres perfiles de resiliencia (Grupo de alta resiliencia, Grupo de baja resiliencia y Grupo de Alta Competencia, Alta Autodisciplina y Baja Aceptación). Posiblemente, por desgracia, los estereotipos y modas que marca la sociedad alejan mucho a las personas con discapacidad de -467 - 
los cánones de la belleza (Matalinares, et al., 2011; Suriá et al., 2015). Esto podría repercutir en unos niveles inferiores de autoestima en el factor de autoconcepto físico.

Por tanto, como los resultados obtenidos reflejan, parece que la resiliencia está relacionada con características que conforman el autoconcepto, y por tanto, ahondar en esta asociación va a favorecer una adecuada adaptación e integración al medio en los jóvenes con discapacidad (Caballo y Verdugo, 2013; Gifré, et al., 2010; Saavedra y Villalta, 2008). No obstante, se deben tener en cuenta algunas limitaciones de este trabajo. La principal radica en que no debemos olvidar que la experiencia de las características de la discapacidad son únicas para cada persona y van a estar conformadas por una compleja combinación de factores (desde las diferencias personales de experiencias, temperamentos y contextos diferentes), que deberían haberse tenido en cuenta a la hora de explicar la variabilidad en los perfiles de resiliencia y en el autoconcepto.

A su vez, es probable que los jóvenes con discapacidad participantes en el estudio, tuvieran más capacidad de superación y estuvieran más motivados para participar en comparación con los participantes reacios a colaborar. De hecho, es lógico suponer que los jóvenes ue acuden a asociaiones de apoyo, sean más propicios a potenciar la resiliencia y el autoconcepto puesto que, pertenecer y asistir a las reuniones en busca de apoyo informativo, práctico o emocional, indica su afán de buscar la superación de sus dificultades. Estos aspectos sobreestimarían el grado de resiliencia y de autoconcepto y podrían sesgar la magnitud de algunas de las asociaciones detectadas. En futuras investigaciones se deberían controlar estos sesgos con objeto de incrementar la validez interna de los resultados.

A pesar de estas limitaciones, se considera que los resultados de este trabajo resultan interesantes al sugerir que, si bien la resiliencia se relaciona con un mejor ajuste de los jóvenes que viven con discapacidad en el desarrollo interpersonal y social, no todas las dimensiones de la resiliencia cobran el mismo peso en este ajuste. De este modo, podría resultar relevante para el diseño y aplicación de programas de entrenamiento y desarrollo de la capacidad resiliente, ya que este patrón lleva a un autoconcepto más elevado entre los jóvenes, por lo que se plantea como objetivo futuro seguir indagando en la relación existente entre la resiliencia de los jóvenes con discapacidad y su autoconcepto. 


\section{Referencias}

Aguado Díaz, A. L. y Alcedo Rodríguez, M. Á. (2012). Necesidades percibidas en el proceso de envejecimiento de las personas con discapacidad intelectual. Siglo Cero, 35(209), 5-19.

Antle, B. J. (2004). Factors associated with self-worth in young people with physical disabilities. Health \& Social Work, 29, 167-175. http://dx.doi.org/10.1093/hsw/29.3.167

Bisquerra-Alzina, R. y Pérez-Escoda, N. (2012). Las competencias emocionales. Educación XX1, 10, 61-82. http://dx.doi.org/10.5944/educxx1.1.10.297

Buscaglia, L. (1990). Los discapacitados y sus padres. Buenos Aires: Emecé.

Caballo, C. y Verdugo, M. Á. (2013). Habilidades sociales. Programa para mejorar las relaciones sociales entre niños y jóvenes con deficiencia visual y sus iguales sin discapacidad. Madrid: ONCE.

Cohen, J. (1988). Statistical Power Analysis for the Behavioral Sciences. New York: Academic Press.

Esnaola, I. (2009). El autoconcepto físico durante el ciclo vital. Anales de Psicología, 24(1), 1-8.

de Boer, A. A., Pijl, S. J. y Minnaert, A. E. M. G. (2010). Attitudes of parents towards inclusive education: A review of the literature. European Journal of Special Needs Education, 25, 165-181. http://dx.doi.org/10.1080/08856251003658694

Floyd, M. H., Zambrano, J. A., Antó, A. M., Jiménez, C. S., Solórzano, C. P. y Díaz, A. L. (2012). Identificación de las barreras del entorno que afectan la inclusión social de las personas con discapacidad motriz de miembros inferiores. Salud Uninorte, 28(2), 112.

Fuentes, M. C., García, J. F., Gracia, E. y Lila, M. (2011). Autoconcepto y ajuste psicosocial en la adolescencia. Psicothema, 23, 7-12. http://dx.doi.org/10.20882/adicciones.148

García, F., Gracia, E. y Zeleznova, A. (2013). Validation of the English version of the fivefactor self-concept questionnaire. Psicothema, 25, 549-555. doi: 10.7334/psicothema2013.33

García, J. F., Musitu, G., Riquelme, E. y Riquelme, P. (2011). A confirmatory factor analysis of the "Autoconcepto Forma 5" questionnaire in young adults from Spain and Chile. The Spanish Journal of Psychology, 14, 648-658. http://dx.doi.org/10.5209/rev_sjop.2011.v14.n2.13

- 469 - $\quad$ Electronic Journal of Research in Educational Psychology, 14(3), 450-473. ISSN: 1696-2095. 2016. no. 40 http://dx.doi.org/10.14204/ejrep.40.15150 
García, F. y Musitu. G. (1999). AF-5. Autoconcepto Forma 5. Madrid: TEA.

Gifre, M., Del Valle, A., Yuguero, M., Gil, Á. y Monreal, P. (2010). La mejora de la calidad de vida de las personas con lesión medular: La transición del centro rehabilitador a la vida cotidiana desde la perspectiva de los usuarios. Athenea Digital, 18, 3-15. http://dx.doi.org/10.5565/rev/athenea.708

Gil-Llario, M.D., Molero-Mañes, R., Ballester-Arnal, R. y Sabater-Pavía, P. (2012). Establecer vínculos afectivos para facilitar la resiliencia de los niños acogidos. Revista INFAD de Psicología, 1, 337-344. http://dx.doi.org/10.1016/j.childyouth.2013.01.013

Gómez-Vela, M., Verdugo, M. Á. y González-Gil, F. (2007). Calidad de vida y autoconcepto en adolescentes con necesidades educativas especiales y sin ellas. Infancia y Aprendizaje, 30, 523-536. http://dx.doi.org/10.1174/021037007782334300

Goñi, E., Fernández-Zabala, A. y Infante, G. (2012). El autoconcepto personal: diferencias asociadas a la edad y al sexo. Aula abierta, 40, 39-50.

Gross-Fava, G. A. y Tomba, E. (2009). Increasing Psychological Well-Being and Resilience by Psychotherapeutic Methods. Journal of Personality, 77, 1903-1934. http://dx.doi.org/10.1111/j.1467-6494.2009.00604.x

Grotberg, E. (1995). A guide to promoting resilience in children: strengthening the human spirit. La Haya: Bernard Van Leer Foundation. Recuperado de: http://resilnet.uiuc.edu/library/grotb95b.html.

Guest, R. S., Klose, K. J., Needham-Shropshire, B. M. y Jacobs, P. L. (1997). Evaluation of a training program for persons with SCI paraplegia using the Parastep ambulation system: Part 4. Effect on physical self-concept and depression. Archives of physical medicine and rehabilitation, 78, 804-807. http://dx.doi.org/10.1016/s0003-9993(97)90191x

Heilemann, M., Lee, K. y Kury, F.S. (2003). Psychometric properties of the Spanish version of the Resilience Scale. Journal Nurs Meas, 11, 61-72. http://dx.doi.org/10.1891/jnum.11.1.61.52067

INE (2008). Encuesta sobre Discapacidad, Autonomía Personal y Situaciones de Dependencia (EDAD). Recuperado

de: http://www.ine.es/jaxi/menu.do?type=pcaxis \&path=/t15/p418=inebase $\& \mathrm{~L}=0$

Juaréz-Sanchez, A., Padilla-Góngora, D. Matínez-Cortes, A.C. López-Liria, R. (2010). Percepción social de la sordera en el entorno escolar: proceso de elaboración de un cuestionario de evaluación. Revista de Logopedia, Foniatría y Audiología, 30(3), 120-129. http://dx.doi.org/10.1016/s0214-4603(10)70159-5

Kotliarenco, M.A. (2000). Actualizaciones en Resiliencia. Buenos Aires: Ediciones UNLA. 
Lacunza, A. B. y de González, N. C. (2011). Las habilidades sociales en niños y adolescentes. $\mathrm{Su}$ importancia en la prevención de trastornos psicopatológicos. Fundamentos en $\mathrm{Hu}$ manidades, 12(23), 159-182.

Luna, N. C. y Molero, D. (2013). Revisión teórica sobre el autoconcepto y su importancia en la adolescencia. Revista Electrónica de Investigación y Docencia (REID), 10, 1-7.

Martínez, F., García, J. M. y Pérez, A. M. (2005). Personalidad y autoconcepto del discapacitado visual. En R. Martínez, P. P. Berruezo, J. M. García y J. Pérez (coords.), Discapacidad visual (pp. 439-455). Desarrollo, comunicación e intervención Granada. Grupo Editorial Universitario.

Matalinares Calvet, M., Arenas, C., Sotelo, L., Sotelo, N., Díaz Acosta, G., Dioses Chocano, A., ... y Tipacti, R. (2013). Factores personales de resiliencia y autoconcepto en estudiantes de primaria de Lima Metropolitana. Revista de Investigación en Psicología, 14, 187-207.

Morales, F. M., Fernández, F. J., Infante, L., Trianes, M. V. y Cerezo, M. T. (2010). Eficacia de una intervención para incrementar apoyo social en adolescentes discapacitados motores a partir del voluntariado de estudiantes de educación secundaria. Revista Latinoamericana de Psicología, 41, 141-152.

Novo-Corti, I., Muñoz-Cantero, J. y Calvo-Porral, C. (2011). Análisis de las actitudes de los jóvenes universitarios hacia la discapacidad: un enfoque desde la teoría de la acción razonada. Revista electrónica de investigación educativa, 17, 1-26. http://dx.doi.org/10.7203/relieve.17.2.4010

OMS (2001). Clasificación Internacional del Funcionamiento de la Discapacidad y de la Salud. Madrid: Ministerio de Trabajo y Asuntos Sociales.

Oshio, A., Kaneko, H., Nagamine, S. y Nakaya, M. (2003). Construct validity of the Adolescent Resilience Scale. Psychological Reports, 93, 1217-1222. http://dx.doi.org/10.2466/pr0.2003.93.3f.1217

Pérez, J. I. y Garaigordobil, M. (2007). Discapacidad motriz: autoconcepto, autoestima y síntomas psicopatológicos. Estudios de Psicología, 28(3), 343-357. http://dx.doi.org/10.1174/021093907782506434

Polo-Sánchez, M. T. y López-Justicia, M. D. (2012). Autoconcepto de estudiantes universitarios con discapacidad visual, auditiva y motora. Revista Latinoamericana de Psicología, 44(2), 87-98. 
Quiceno, J. M. y Vinaccia, S. (2012). Resiliencia y características sociodemográficas en enfermos crónicos. Psicología desde el Caribe, 29, 87-104.

Rey, L. y Extremera, N. (2012). Inteligencia emocional percibida, felicidad y estrategias distractoras en adolescentes. Boletín de psicología, 104, 87-101.

Rodríguez, M., Pereyra, M. G., Gil, E., Jofré, M., De Bortoli, M. y Labiano, L. M. (2009). Propiedades psicométricas de la escala de Resiliencia versión argentina. Revista Evaluar, 9, 72-82.

Rybarczyk, B., Emery, E. E., Guequierre, L. L., Shamaskin, A. y Behel, J. (2012). The Role of Resilience in Chronic Illness and Disability in Older Adults. Annual Review of Gerontology \& Geriatrics, 32, 173-188. http://dx.doi.org/10.1891/0198-8794.32.173

Rutter, M. (1972). Maternal Deprivation Reassessed. Harmondsworth: Penguin.

Saavedra, E. y Villalta, M. (2008). Estudio comparativo en Resiliencia con sujetos discapacitados motores. Revista Investigaciones en Educación, 8, 81-90.

Salgado, A. C. (2005). Métodos e instrumentos para medir la resiliencia: una alternativa peruana. Liberabit, 11, 41-48.

Sánchez, M. T. P. y López-Justicia, M. D. (2012). Autoconcepto de estudiantes universitarios con discapacidad visual, auditiva y motora. Revista Latinoamericana de Psicología, 44, 87-98.

Schalock, R. L. (2013). La nueva definición de discapacidad intelectual, apoyos individuales y resultados personales. Siglo Cero, 40(229), 22-39.

Sherer, M., Maddux, J. E., Mercandante, B., Prentice-Dunn, S., Jacobs, B. y Rogers, R. W. (1982). The self-efficacy scale: Construction and validation. Psychological reports, 51, 663-671. http://dx.doi.org/10.2466/pr0.1982.51.2.663

Silva-Dreyer, C. L. y Martínez-Guzmán, M. L. (2007). Empoderamiento, participación y autoconcepto de persona socialmente comprometida en adolescentes chilenos. Interamerican Journal of Psychology, 41, 129-138. http://dx.doi.org/10.4067/s071822282004000200003

Suriá, R., García-Fernández, J.M. y Ortigosa, J. (2015). Perfiles resilientes y su relación con las habilidades sociales en personas con discapacidad motora. Psicología Conductual, 23, 35-49. http://dx.doi.org/10.1016/j.gaceta.2015.01.016

Suriá, R. (2014). Actitudes integradoras en el contexto educativo de los estudiantes con discapacidad según la percepción de sus progenitores. Bordón. Revista de Pedagogía, 66, 157-172. http://dx.doi.org/10.13042/bordon.2014.66410 
Suriá, R. (2014).Discapacidad motora y resilencia análisis en función de la edad, grado y etapa en la que se adquiere la discapacidad. Siglo Cero: Revista Española sobre Discapacidad Intelectual, 45, 6-18.

Suriá R. (2012). ¿Se sienten integrados los estudiantes con discapacidad en su centro educativo?: análisis en función del tipo de discapacidad y etapa formativa. Profesorado: Revista de curriculum y formación del profesorado, 16, 341-356.

Thompson, J. R., Bradley, V. J., Buntinx, W. H., Schalock, R. L., Shogren, K. A., Miller, M. E. S., ... y Gómez, S. C. (2010). Conceptualizando los apoyos y las necesidades de apoyo de personas con discapacidad intelectual. Siglo Cero: Revista Española sobre Discapacidad Intelectual, 41(233), 7-22.

Uriarte, J.D. (2013). La perspectiva comunitaria. Psicología Política, 47, 7-18.

Vara, M. C. R. y Rodríguez, J. M. A. (2011). Validación psicométrica de la escala de resiliencia en una muestra de adolescentes portugueses. Psicopatología Clínica, Legal y Forense, 11, 51-65.

Wagnild, G.M. y Young, H.M. (1993). Development and psychometric evaluation of the Resilience Scale. Journal Nurs Meas, 1, 165-78. 